\title{
Study on the Origin of Fluorescence by using dual-emission Carbon
}

\section{Dots}

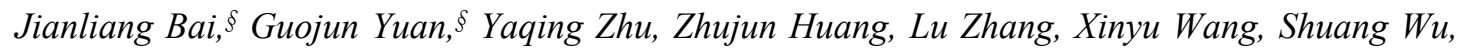
Lili Ren*

School of Chemistry \& Chemical Engineering, Southeast University, Nanjing 211189, China

[*] Corresponding author. E-mail address: liliren@seu.edu.cn (L. Ren).

[§] These authors contributed equally to this work. 


\section{Instrumentation}

Transmission Electron Microscopy (TEM) and high-resolution transmission electron microscopy (HR-TEM) figures were obtained on a JEM-2100 microscope operating at $200 \mathrm{kV}$. X-ray photoelectron spectroscopy (XPS) was carried out with Thermo ESCALAB 250XI instrument. The Fourier transform infrared spectra (FT-IR) were measured on a Bruker Tensor 27 ATR-FTIR spectrometer using $\mathrm{KBr}$ pellet. Fluorescence (FL) spectra values were collected on a Horiba Fluoromax-4 fluorescence spectrophotometer. UV-Vis absorption spectra were recorded on a Shimadzu UV-2600 spectrometer. ${ }^{1} \mathrm{H}$ NMR spectra were recorded on a Bruker AVANCE 600 spectrometer.

\section{Synthesis route of $C D s(F A)$}

The CDs (FA) in our work were prepared using the method previously reported literature [1]. Briefly, folic acid (FA) (0.25 g) was dissolved DI water (25 ml). After stirring for mixing, the solution was transferred to flanged stainless steel reactor liner $(50 \mathrm{~mL})$ and heated at $260{ }^{\circ} \mathrm{C}$ for $2 \mathrm{~h}$. After the reaction, the reactors were cooled to room temperature naturally. The obtained dark brown solution was centrifuged under at high speed $\left(10000 \mathrm{rad} \mathrm{min}^{-1}\right)$ for $20 \mathrm{~min}$ to remove large or agglomerated particles. Purely luminescent CDs were obtained via freeze drying. The obtained CDs were then used to make stock solution $(0.5 \mathrm{mg} / \mathrm{ml})$ for further use.

\section{Synthesis route of Poly-PPD-dots}


$0.020 \mathrm{~g}$ PPD was dissolved in $10 \mathrm{~mL}$ of deionized water and stirred for about $10 \mathrm{~min}$. The mixed solution was transferred to a $20 \mathrm{~mL}$ Teflon-lined stainless-steel autoclave and heated at $200{ }^{\circ} \mathrm{C}$ for $2 \mathrm{~h}$. After the reaction was complete, a cloudy solution with a small amount of precipitate was obtained, which was purified by silica column chromatography using ethanol as the eluent.

\section{References}

(1) Li, Q.; Zhou, M.; Yang, Q.; Wu, Q.; Shi, J.; Gong, A.; Yang, M.; Efficient Room-Temperature Phosphorescence from Nitrogen-Doped Carbon Dots in Composite Matrices. Chem. Mater. 2016, 28, 8221-8227. 


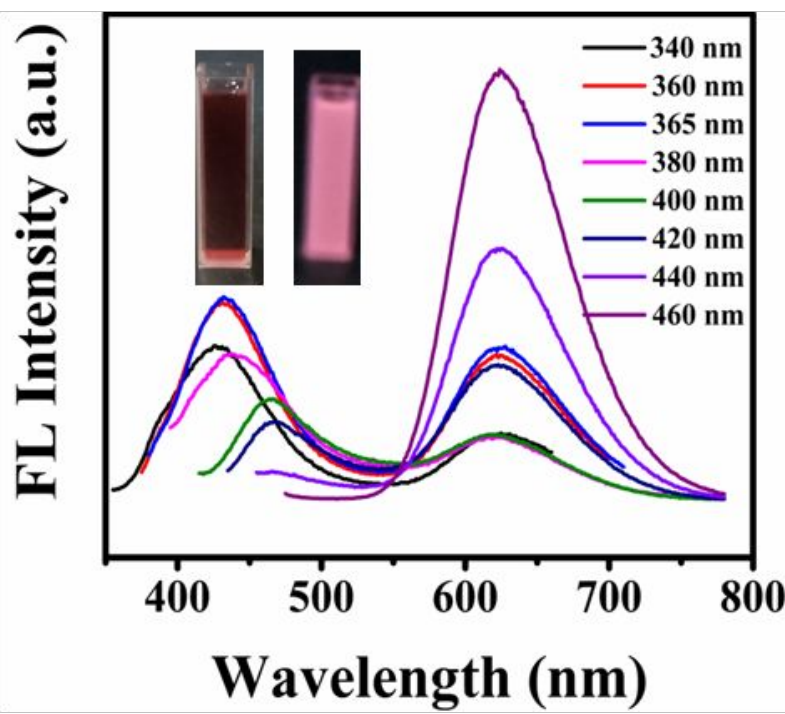

Figure S1 The FL spectra of N-CDs excited by different wavelength lights in $\mathrm{H}_{2} \mathrm{O}$. Insert: images of N-CDs under sunlight (left) and UV lamp (right). 
(a)

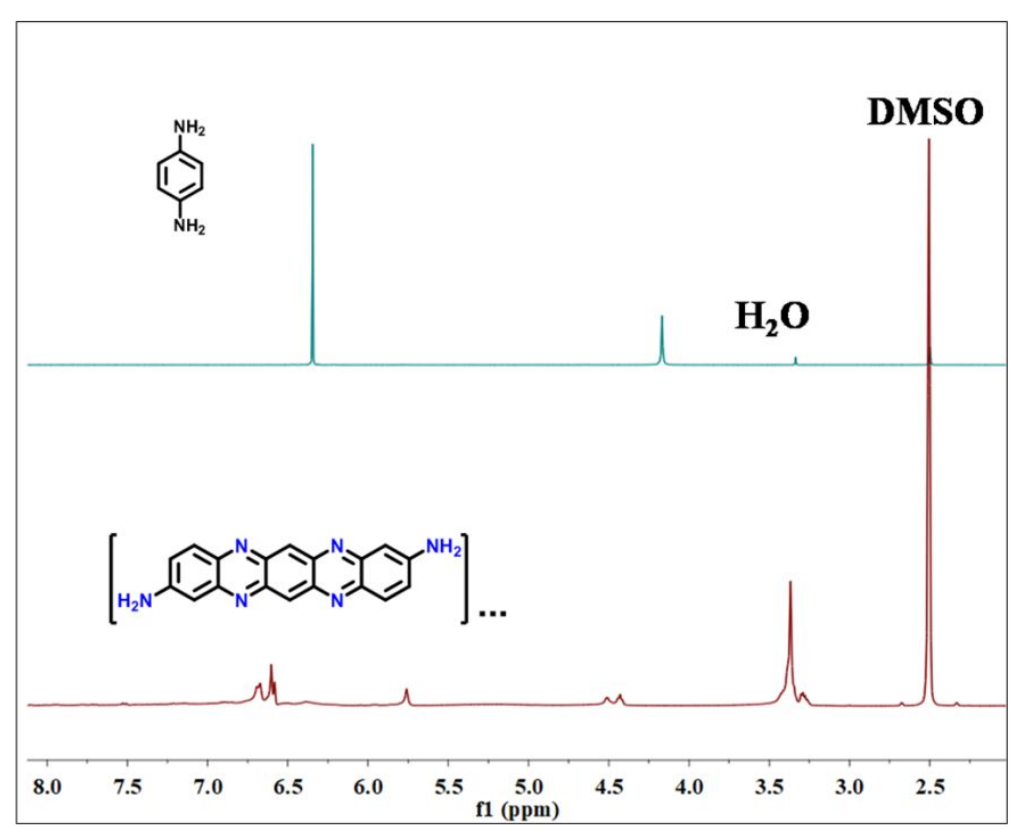

(b)

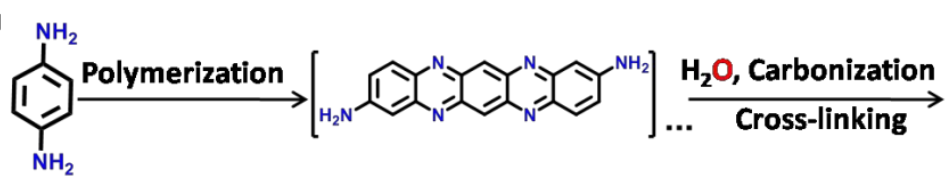

Poly-PPD-dots

Figure S2 (a) ${ }^{1} \mathrm{H}$ NMR spectra of PPD and Poly-PPD-dots in DMSO-d 6 . (b) A plausible formation process and structure of the Poly-PPD-dots. 


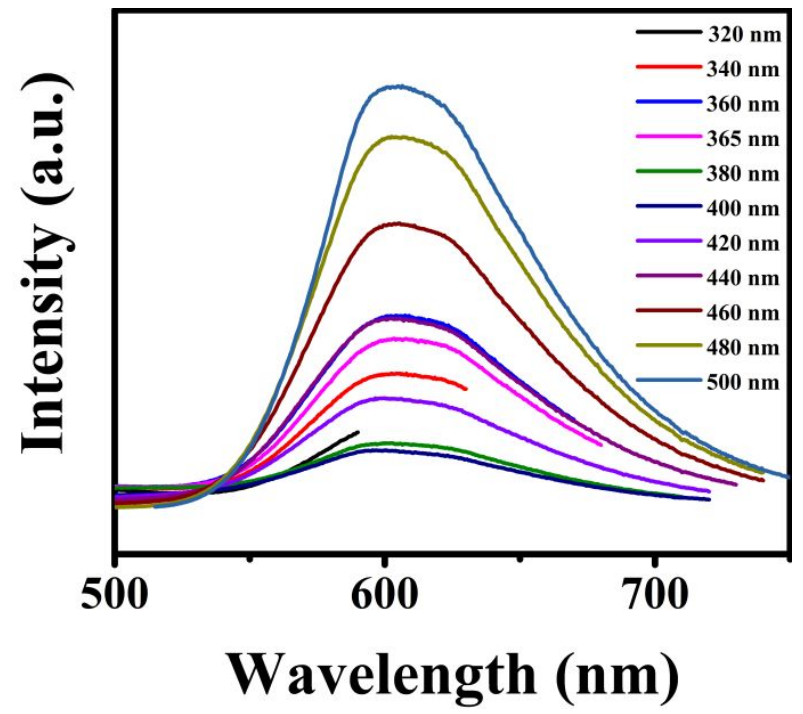

Figure S3 The FL spectra of Poly-PPD-dots excited by different wavelength lights in ethanol. 


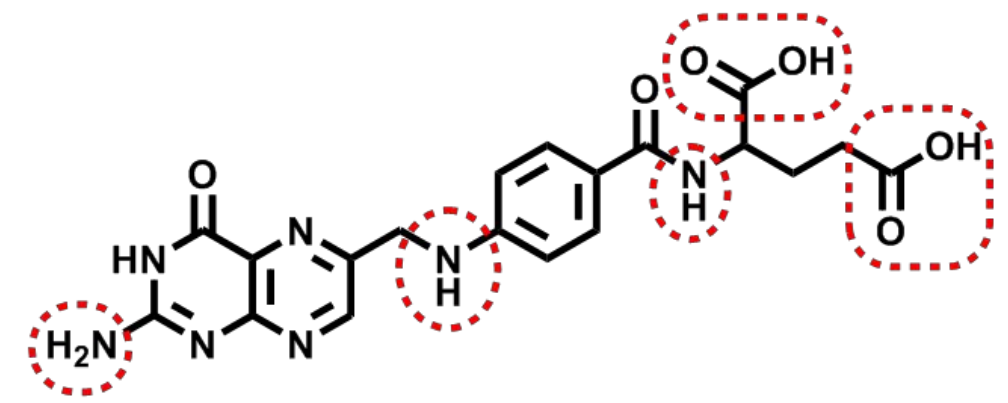

Figure S4 Folic acid (FA) molecule contains many sites of dehydration. 


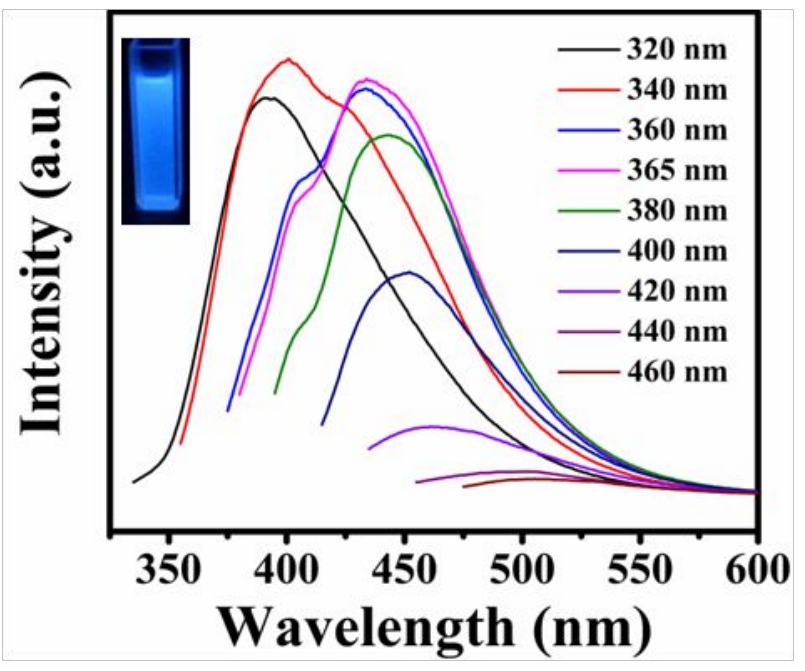

Figure S5 The FL spectra of CDs (FA) excited by different wavelength lights in $\mathrm{H}_{2} \mathrm{O}$. Insert: images of CDs (FA) under UV lamp. 